\title{
THE EFFECTIVE TEMPERATURE OF JUPITER'S EQUATORIAL BELT DURING THE 1965 APPARITION
}

\author{
L. M. TRAFTON \\ University of Texas at Austin, Austin, Tex., U.S.A. \\ and \\ R. WILDEY \\ U.S. Geological Survey, Center of Astrogeology, Flagstaff, Ariz., U.S.A.
}

\begin{abstract}
Preliminary results from our study of Jupiter's equatorial limb darkening at 8-14 $\mu$ indicate that the effective temperature of the equatorial belt lay between $140 \mathrm{~K}$ and $150 \mathrm{~K}$. We find these temperatures to be insensitive to the likely range of the $\mathrm{He} / \mathrm{H}_{2}$ ratios. These results assume that Jupiter's only significant sources of extinction at these wavelengths are the pressureinduced absorption of $\mathrm{H}_{2}$ and $\mathrm{He}$ and the absorption of saturated $\mathrm{NH}_{3}$.
\end{abstract}

Limb-darkening observations of Jupiter's equatorial belt in the 8-14 $\mu$ spectral region were obtained at various times throughout the 1965 apparition. They consisted of diametric scans in right ascension and were collected from the 200-inch Hale telescope with the aid of a $\mathrm{Hg}$-doped $\mathrm{Ge}$ photoconductor cooled with liquid $\mathrm{H}_{2}$. The apparatus and technique have been described previously (Wildey, 1966 and 1968).

The diameter of the aperture was one eighth of Jupiter's apparent radius. The angle between a given scan and the Jovian equator averaged out to be only a few degrees.

We reduced the observations to obtain a mean limb-darkening profile corresponding to unit atmospheric thickness. The two points of each scan where the signal merged with the noise defined the end points of an abscissa having 40 equal intervals. We scaled the ordinate to provide unit area for the portion of each scan profile lying above the abscissa.

Let $S_{i j}$ be the logarithm of the ordinate defined at the $j$ th division of the abscissa and for the $i$ th scan. For a given $j$, we evaluated the mean of $S_{i j}$ over all the scans and fitted a least squares quadratic polynomial in $\sec Z$ to the difference between this mean and its corresponding set of ordinates $S_{i j}(i=1, \ldots, 41)$. We then computed the residual between each of these ordinates and the corresponding polynomial values and eliminated scan points whose residual was greater than 2.5 times the mean residual. Using the remaining scan points, we repeated this procedure, iterating until no more scan points were rejected. We did this for 38 values $(j=2, \ldots, 39)$ and used the resulting set of polynomials to generate a mean $\operatorname{limb}$ darkening profile at $\sec Z=1$. Three cases retained all 41 scan points and one case had as few as 29 of the original 41 scan points surviving the iteration. This profile differed negligibly from the mean limb darkening profile. The statistical accuracy of each point of the final profile was better than one percent. This profile is illustrated in Figure 1.

In order to interpret this profile, we constructed non-gray model atmospheres for Jupiter in hydrostatic and thermal equilibrium (Trafton, 1967). The pressure-induced absorption of $\mathrm{H}_{2}$ and $\mathrm{He}$ served as the thermal opacity. We added saturated $10 \mu$ and $16 \mu \mathrm{NH}_{3}$ bands to the models but neglected the opacity of the rotational band of 


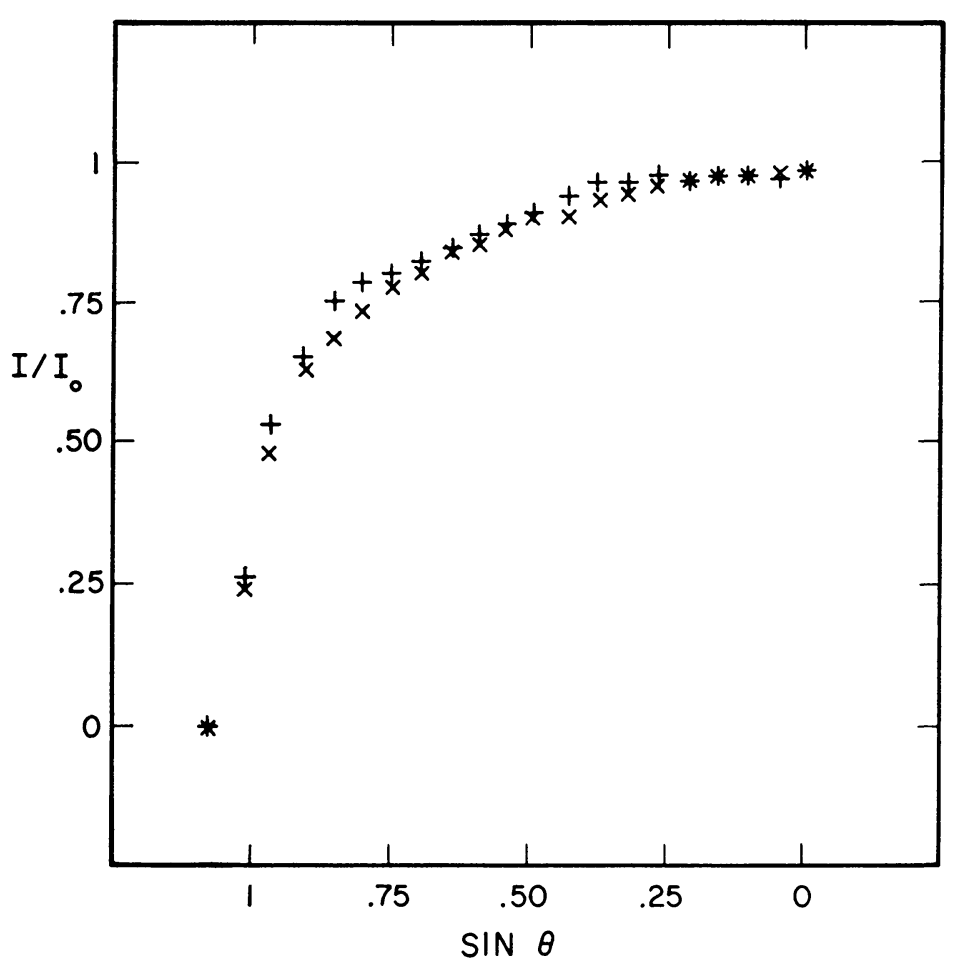

Fig. 1. The reduced observational limb-darkening profile corresponding to unit atmospheric thickness. The pluses denote the sunrise limb and the crosses denote the sunset limb.

$\mathrm{NH}_{3}$ and the extinction from any particle scattering in the upper Jovian atmosphere. Most of the 8-14 $\mu$ radiation comes predominantly from layers in Jupiter's atmosphere at a Planck mean optical depth near unity. The effective temperature $\left(T_{e}\right)$ and $\mathrm{He} / \mathrm{H}_{2}$ ratio were left as free parameters to be determined by fitting a family of models to the observed limb darkening curves.

The shape of the limb darkening curve depends sensitively on the relative concentration of $\mathrm{H}_{2}$ and $\mathrm{NH}_{3}$ because of the different dependences on pressure and temperature. Because we assume $\mathrm{NH}_{3}$ to be saturated in Jupiter's upper atmosphere, this relative concentration is sensitive to the boundary temperature and, therefore, to the effective temperature. Using limb darkening observations to deduce $T_{e}$ requires a reliable band model for $\mathrm{NH}_{3}$.

We constructed a model for the $10 \mu$ and $16 \mu \mathrm{NH}_{3}$ bands by the following procedure: We measured the equivalent widths and recorded the wavelengths of 550 lines from the published high resolution data of Garing et al. (1959) and Mould et al. (1959). Using the curve of growth theory of Ladenburg and Reiche (1913) for lines having Lorentz cores and assuming we could approximate all the lines to have the same half width, we derived line strengths for these bands. Most of the $10 \mu$ laboratory lines were saturated.

Most of the radiation passes between the lines so that the mean transmission of the 
band is very sensitive to the shape of the lines in the wings except when the mean transmission is large. For the line shape in the wings we assumed a power law dependence on the frequency difference from the line center and determined this dependence by fitting the $\mathrm{NH}_{3}$ band model to the low resolution data of France and Williams (1966). Their data consisted of $\mathrm{NH}_{3}$ band absorptance measurements between 660 and $1300 \mathrm{~cm}^{-1}$ over many decades of path length and effective pressure, all obtained at room temperature. We found that the Lorentz line shape was invalid for the wings of $\mathrm{NH}_{3}$. This shape would not allow the high resolution band model to fit the low resolution band data over the entire domain of laboratory pressures and path lengths. On the other hand, we did find a special power-law shape which caused better agreement. It falls off more slowly in the wing (as the 1.78 power of the frequency difference) than does the Lorentz shape. This indicates the sensitivity of line shape in the wings to the intermolecular potential.

After having established this dependence we were then forced by the absence of laboratory data to assume that essentially this same shape holds when $\mathrm{NH}_{3}$ is broadened primarily by collisions with $\mathrm{H}_{2}$ and $\mathrm{He}$ molecules rather than by other $\mathrm{NH}_{3}$ molecules. This is the dominant mechanism for broadening $\mathrm{NH}_{3}$ in Jupiter's upper atmosphere. Using laboratory data for the $\mathrm{NH}_{3}$ line broadening coefficients for $\mathrm{H}_{2}$ and He (Howard and Smith, 1950; Giver, 1968) helped to offset this uncertainty. The pressure dependence had to be derived approximately from consideration of the intermolecular potential (Townes and Schawlow, 1955).

We converted the line strengths to $130 \mathrm{~K}$, the approximate temperature of the levels of formation of the observed 8-14 $\mu$ radiation, by altering the Boltzmann factors and partition function. We left the half widths expressed as a function of the local temperature. The effect of this was to reduce the $16 \mu$ band strength by a factor of 200 and to narrow the $10 \mu$ band by approximately $30 \%$.

We computed a table of the mean transmission of these bands vs path length and pressure for homogeneous paths by integrating the monochromatic transmission over a frequency grid fine enough to delineate the band structure even at low pressures. These mean transmissions were evaluated at intervals of 10 wave numbers.

In order to determine the effective pressure and path length corresponding to the inhomogeneous path overlying a given atmospheric layer, we generalized the CurtisGodson approximation to apply to the non-Lorentzian line shape. These parameters give the same mean transmission in both the weak band and strong band limits along a homogeneous path as along the actually inhomogeneous path. We evaluated these parameters for each layer of each model. Interpolation in the table of mean transmissions provided the values of the mean transmissions $T_{v}\left(\mathrm{NH}_{3}\right)$ corresponding to the absorption of saturated $\mathrm{NH}_{3}$ along the atmospheric path at direction cosine $\mu$. Figure 2 illustrates the mean transmission of $10 \mathrm{~cm}$-atm of $\mathrm{NH}_{3}$ thinly diluted in 0.48 and $1.92 \mathrm{~atm}$ of $\mathrm{H}_{2}$, respectively.

The expression for the limb darkening is

$$
I_{v}(\mu)=\int_{0}^{\infty} B_{v}\left(\tau_{v}\right) \exp \left[-\frac{\tau_{v}\left(\mathrm{H}_{2}+\mathrm{He}\right)}{\mu}\right] T_{v}\left(\mathrm{NH}_{3}\right) \frac{\mathrm{d} \tau_{v}}{\mu},
$$


where the subscript $v$ denotes an average value over a 10 wave-number interval evaluated at $V$. We calculated limb darkening profiles for families of $T_{e}$ and $\mathrm{He} / \mathrm{H}_{2}$, doing the integrations to depths of $\tau_{v}>12$. An exponential quadrature assured numerical accuracy. We weighted these curves by the spectral response of the instrument and by the mean atmospheric spectral transmission. After integrating over frequency, we convoluted the limb darkening signals with the Gaussian seeing function for values of the seeing in the neighborhood of $3^{\prime \prime}$ arc. We also folded in the semi-elliptical function for the circular aperture of the instrument. We weighted Jupiter's apparent diameter by the number of scans obtained at that diameter to obtain a mean value for scaling the aperture. We then normalized the profiles to unit central intensity. The shape of the curve is sensitive to $T_{e}$ but is relatively insensitive to fluctuations in the atmospheric transmission.

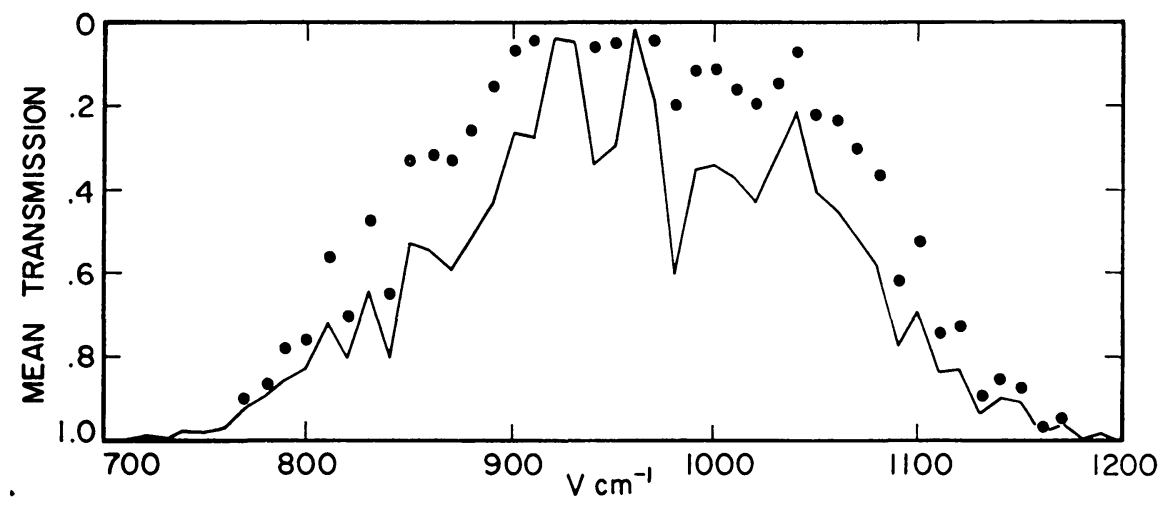

Fig. 2. The mean transmission over intervals of 10 wave numbers for the model $10 \mu$ and $16 \mu$ $\mathrm{NH}_{3}$ bands. The points correspond to $10 \mathrm{~cm}$-atm of $\mathrm{NH}_{3}$ thinly diluted in 0.48 and $1.92 \mathrm{~atm}$ of $\mathrm{H}_{2}$, respectively, at $130 \mathrm{~K}$.

We checked our assumption that the omission of Jupiter's rotational $\mathrm{NH}_{3}$ band from the models would not significantly alter the shape of the limb darkening profile by inserting a very schematic model for this band and letting the model atmosphere relax to radiative equilibrium. The band model simply absorbed at the same depths and frequencies as the rotational $\mathrm{NH}_{3}$ band. Calculating theoretical profiles by the above procedure, we found the difference to be small compared with that resulting from varying the effective temperature by $10 \mathrm{~K}$. The effect of varying the $\mathrm{He} / \mathrm{H}_{2}$ ratio for values less than unity was also small.

To compare the observational and theoretical profiles, we normalized the former to unit central intensity and calibrated their abscissae in terms of $\sin \theta$ by equating their areas to the corresponding theoretical profiles.

Figure 3 illustrates the comparison for two temperatures. The models include the schematic $\mathrm{NH}_{3}$ rotational band and assume $\mathrm{He} / \mathrm{H}_{2}=0$. The shaded region shows the $T_{e}=150 \mathrm{~K}$ model limb-darkening for extreme values of the seeing; namely, $1^{\prime \prime}$ to $5^{\prime \prime}$ arc. Note the rather small effect of the seeing. The crosses denote the corresponding observations for the sunset limb. The solid line shows the $T_{e}=130 \mathrm{~K}$ model for seeing 


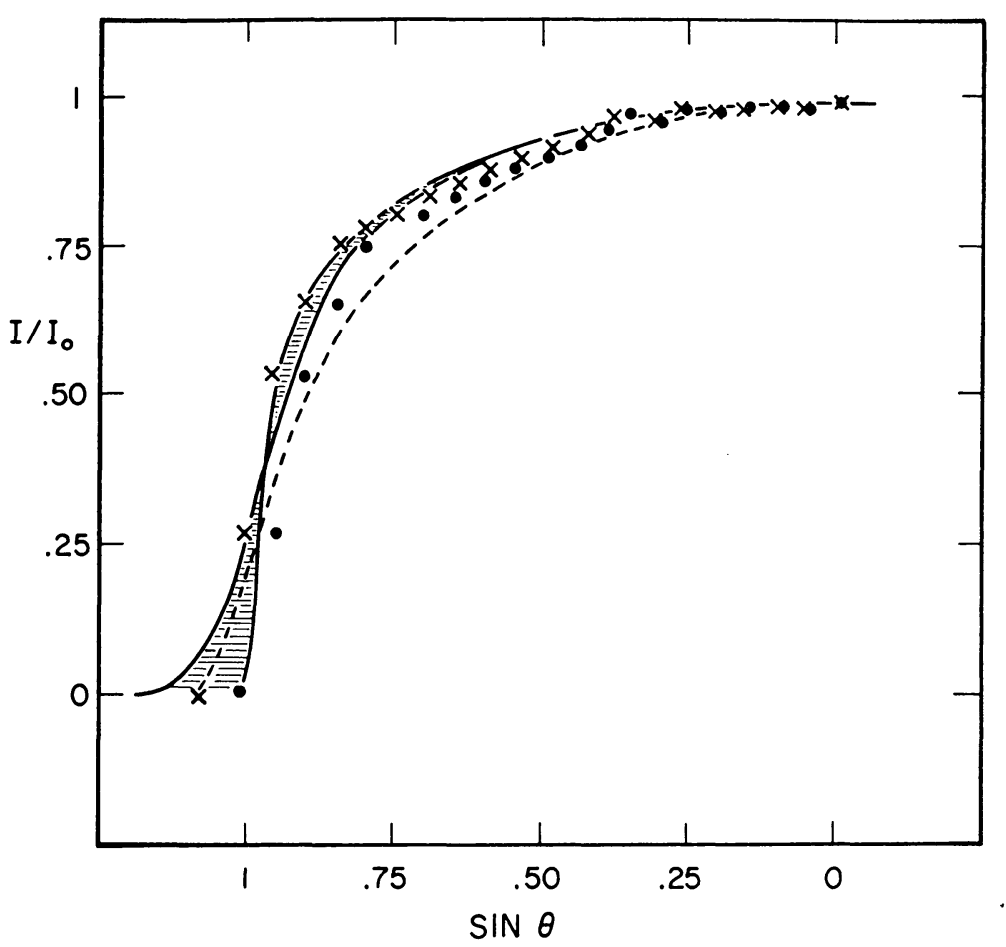

Fig. 3. Comparison of observational and theoretical profiles. The shaded region denotes the $T_{e}=150 \mathrm{~K}$ curves calculated for values of the seeing between $1^{\prime \prime}$ and $5^{\prime \prime}$ arc. The crosses are the correspondingly normalized and reduced observational data. The $T_{e}=130 \mathrm{~K}$ comparison is illustrated for seeing $3^{\prime \prime}$ arc; the dots represent the observational data and the dashed line represents the model's profile.

equal to $3^{\prime \prime}$ arc and the dots are the correspondingly normalized observations. Observe that the fit is distinctly better for the $T_{e}=150 \mathrm{~K}$ model. Varying $\mathrm{He} / \mathrm{H}_{2}$ causes only a minor effect. Our preliminary results indicate that the effective temperature of Jupiters' equatorial belt lies between 140 and $150 \mathrm{~K}$.

In principle, we can extend this procedure to derive a $\mathrm{He} / \mathrm{H}_{2}$ ratio by examining the magnitude of the signal from the center of the disk. Whether the uncertainties in our knowledge of the earth's atmospheric transmission and the schematic rotational $\mathrm{NH}_{3}$ band will permit us to derive improved limits on the $\mathrm{He} / \mathrm{H}_{2}$ ratio remains for our final analysis to reveal.

\section{References}

France, W. L. and Williams, D.: 1966, J. Opt. Soc. Amer. 56, 70.

Garing, J. S., Nielson, H. H., and Rao, K. N.: 1959, J. Molecular Spectrosc. 3, 496.

Giver, L.: 1968, private communication.

Howard, R. and Smith, W. V.: 1950, Phys. Rev. 79, 132.

Landenburg, R. W. and Reiche, F.: 1913, Ann. Phys. 42, 181.

Mould, H. M., Price, W. C., and Wilkinson, G. R.: 1959, Spectrochim. Acta 15, 314. 
Townes, C. H. and Schawlow, A. L.: 1955, in Microwave Spectroscopy, New York, Chapters 12-13.

Trafton, L. M.: 1967, Astrophys. J. 147, 765.

Wildey, R. L.: 1966, Z. Astrophys. 64, 32.

Wildey, R. L.: 1968, Astrophys. J. 154, 761.

\section{Discussion}

Pollack: What did you say your estimated absolute temperature was and how do you interpret it? Trafton: Between 140 and $150 \mathrm{~K}$; this is the temperature which when raised to the fourth power and multiplied by $\sigma$, is equal to the total flux which departs from the planet.

Ingersoll: Did you investigate the dependence of this derived effective temperature on the various free parameters in your model?

Trafton: Yes, as illustrated in the second slide. The two main free parameters were the effective temperature and the helium-to-hydrogen ratio. The derived shape of the limb darkening curve depends very sensitively on the effective temperature but only insensitively on the helium/hydrogen ratio. We can bracket the latter from observations to lie between zero and unity; this is the main reason for the insensitivity arising from the latter.

Ingersoll: Did you have free parameters in preparing the line profile? And what about the ammonia itself; is that a free parameter, and how did you choose it?

Trafton: The only parameters describing the line profile were the effective temperature and the helium/hydrogen ratio; we assumed the ammonia was saturated in the atmosphere. If it turns out that ammonia is not saturated, then of course our results would not be valid. However, the close agreement between the first and final slide in the series would tend to indicate that the situation probably is not too different from what we expect it to be.

Pollack: If you allow for a slight deviation, then maybe the $130 \mathrm{~K}$ curve would give a better fit?

Trafton: No. The effective temperature is very sensitive to the relative absorption of the pressureinduced opacities and ammonia, because of their very different pressure and temperature dependence upon Jupiter's depth. The shape of the limb darkening curves is sensitive to the effective temperature.

Stephens: You say that temperatures correspond to unit optical depth. What sort of number densities would this correspond to?

Trafton: I would guess that this might be close to a third of an atmosphere. This absorption takes place relatively high in Jupiter's atmosphere, well above the cloud layers, and well into the radiative zone. 